\title{
A randomised controlled study: the effects of internal sphincterotomy of the anus on defecation disorders in patients with outlet obstructive and mixed constipation
}

\author{
Chengguo Bin, Daiping Gong, Yanling He \\ Anorectal Department, The People's Hospital of Dazu, Chongqing, China \\ Contributions: (I) Conception and design: C Bin; (II) Administrative support: C Bin; (III) Provision of study materials or patients: C Bin; (IV) \\ Collection and assembly of data: D Gong, Y He; (V) Data analysis and interpretation: C Bin; (VI) Manuscript writing: All authors; (VII) Final \\ approval of manuscript: All authors. \\ Correspondence to: Chengguo Bin. Department of Anorectal, The People's Hospital of Dazu, No.138 Longgang West Street, Dazu District, \\ Chongqing 402360, China. Email: 1047429335@qq.com.
}

Background: This study sought to investigate the effects of internal sphincterotomy of the anus in the treatment of defecation disorders in patients with outlet obstructive and mixed constipation.

Methods: This study included 250 patients, who met the Rome III criteria for functional defecation disorder-associated constipation and were admitted to the Proctology Department in the People's Hospital of Dazu District between July 2014 and June 2016. The patients were randomly divided into the traditional medical treatment group $(n=125)$ and the surgical treatment group $(n=125)$, and received traditional treatment and internal anal sphincterotomy, respectively. All patient clinical data were retrospectively analyzed. Indices relating to anorectal pressure, electromyographic indices of abdominal muscles, sleep quality, anxiety and depression, defecography and anal healing before and after treatment were measured, and the effects of the different treatment modalities on defecation disorders were analyzed.

Results: The total treatment effective rate was significantly higher in the surgical treatment group than in the traditional treatment group $(\mathrm{P}<0.05)$. The Constipation Scoring System scores at time points 1,2 and 3 were significantly lower in the surgical treatment group than in the traditional treatment group $(\mathrm{P}<0.001)$. After treatment, the Hospital Anxiety and Depression Scale and Pittsburg Sleep Quality Index scores were significantly lower in the surgical treatment group than in the traditional treatment group $(\mathrm{P}<0.001)$. After treatment, the rectal resting pressure and 10SR were significantly lower in the surgical treatment group than in the traditional treatment group $(\mathrm{P}<0.001)$. After treatment, the negative conversion rate of the balloon expulsion test was significantly higher in the surgical treatment group than in the traditional treatment group $(\mathrm{P}<0.05)$, while the rectocele, perineum descending distance, and anorectal angle of the efforts phase were significantly lower in the surgical treatment group than in the traditional treatment group $(\mathrm{P}<0.001)$.

Conclusions: Internal anal sphincterotomy can significantly relieve defecation disorders and clinical symptoms, improve sleep quality, eliminate psychological disorders, and promote patient recovery.

Trial Registration: Chinese Clinical Trial Registry ChiCTR2100048818.

Keywords: Internal sphincterotomy of the anus; outlet obstructive constipation; mixed constipation; defecation disorder; efficacy analysis

Submitted Apr 08, 2021. Accepted for publication Aug 16, 2021.

doi: 10.21037/apm-21-1190

View this article at: https://dx.doi.org/10.21037/apm-21-1190 


\section{Introduction}

Constipation, which is defined as difficulty in defecation, is a high-incidence gastroenterological disorder. It mainly manifests as reduced defecation frequency and dry and hard stools which are difficult to pass $(1,2)$. Chronic constipation can be caused by multiple functional and organic diseases including functional defecation disorders, constipationpredominant irritable bowel syndrome, and functional constipation. Dyssynergic defecation, a common pathological type of chronic constipation, is specifically classified into outlet obstructive constipation and mixed constipation.

Some foreign scholars believe that the leading pathogenesis of constipation is an inability of the rectal and anal sphincters to coordinate their movement. Therefore, at present, the primary aim of treatment for chronic constipation is the restoration of defecation function and intestinal motility $(3,4)$. Currently, the treatment of chronic functional constipation mainly relies on medical and surgical treatments, with the former including drug therapy, biological feedback, dietary intervention, and psychological adjustment, and the latter including rectal prolapse and rectocele surgery. However, these 2 treatment approaches are still far away from having the ideal clinical efficacy. Internal sphincterotomy of the anus can effectively relieve the symptoms of chronic constipation based on short-term observation of clinical symptoms. However, neither indepth study of changes in pre- and postoperative-related clinical indices, nor accurate data have illuminated the specific role of this procedure in clinical treatment (5-7). To address this, the present study set out to analyze the effects of internal sphincterotomy of the anus in the treatment of defecation disorders in patients with outlet obstructive and mixed constipation. We present the following article in accordance with the CONSORT reporting checklist (available at https://dx.doi.org/10.21037/apm-21-1190).

\section{Methods}

\section{General information}

The study was a two-parallel study. The allocation ratio is 1:1. A total of 250 patients, who met the Rome III criteria for constipation caused by functional defecation disorders and were admitted to the Proctology Department in the People's Hospital of Dazu District (Chongqing, China) between July 2014 and June 2016, were enrolled. The patients were divided into 2 groups, traditional medical treatment group $(\mathrm{n}=125)$ and surgical treatment group ( $\mathrm{n}=125)$, according to the order of admission. The traditional medical treatment group comprised 75 males and 50 females, who had an average age of $(46.72 \pm$ 6.72 ) years old and an average disease course of (3.28 1.26) years; the surgical treatment group consisted of 78 males and 47 females, who had an average age of $(46.69 \pm 6.69)$ years old and an average disease course of $(3.30 \pm 1.27)$ years. The clinical data of the patients in both groups were retrospectively analyzed. No significant differences were found in clinical information between the 2 groups $(\mathrm{P}>0.05)$, meaning the 2 groups were comparable (Table 1 and Figure 1).

\section{Inclusion criteria}

To be included in the study, patients needed to meet the following criteria: (I) great effort required in at least $25 \%$ of defecation attempts; (i) dry or hard stools accounting for at least $25 \%$ of bowel movements; (ii) the sense of incomplete evacuation after defecation at least $25 \%$ of the time; (iii) anorectal obstruction and blockage occurring in at least $25 \%$ of defecation attempts; (iv) manual assistance (e.g., assistance with fingers or pelvic floor support) required for defecation at least $25 \%$ of the time; (v) infrequent defecation (fewer than 3 times per week). (II) Patient seldom had loose stools when laxatives were not used. (III) Patient did not meet the diagnostic criteria for irritable bowel syndrome. (IV) During repeated attempts to defecate, the patient met at least 2 of the following 3 criteria: (i) diminished defecation confirmed by balloon expulsion test or imaging examination; (ii) a diagnosis of absonant contraction of the pelvic floor muscles (e.g., the sphincter ani or puborectalis), or a resting pressure and relaxation rate of the sphincter of less than $20 \%$, as determined by manometry, imaging examination, or electromyography; (iii) a diagnosis of poor rectal propulsion during defecation by manometry or imaging examination (Figure 1).

This study was approved by the ethics committee of the People's Hospital of Dazu (Chongqing, China) (No.20140456), and all patients signed informed consent. All procedures performed in this study involving human participants were in accordance with the Declaration of Helsinki (as revised in 2013).

\section{Exclusion criteria}

The following patients were excluded from the study: (I) patients with constipation caused by organic diseases 
Table 1 Comparison of general data in the 2 groups of patients [n (\%), $\bar{x} \pm \mathrm{s}$ ]

\begin{tabular}{|c|c|c|c|c|}
\hline Items & Research group $(n=125)$ & Reference group $(n=125)$ & $\chi^{2} / t$ & $P$ \\
\hline Male & $62(49.60 \%)$ & $66(52.80 \%)$ & & \\
\hline Female & $63(50.40 \%)$ & $59(47.20 \%)$ & & \\
\hline Average age (years old) & $47.33 \pm 9.16$ & $49.19 \pm 9.32$ & 1.59 & 0.11 \\
\hline Smoking history & & & 0.02 & 0.90 \\
\hline No & $70(56.00 \%)$ & $69(55.20 \%)$ & & \\
\hline Yes & $55(44.00 \%)$ & $56(44.80 \%)$ & & \\
\hline Drinking history & & & 0.26 & 0.61 \\
\hline
\end{tabular}

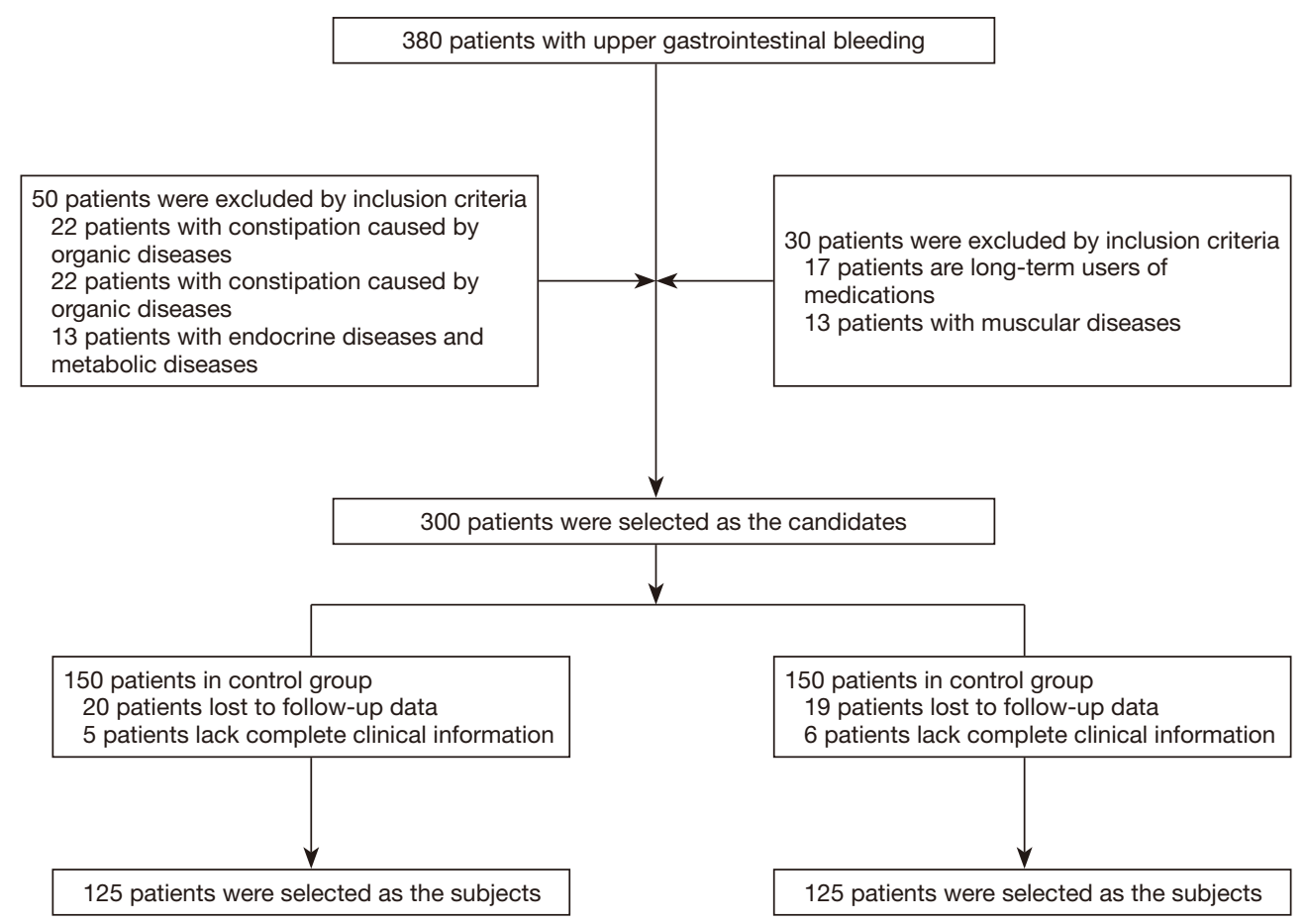

Figure 1 The flow diagram of subject selection.

or drugs; (II) patients with systemic organic diseases in the heart, liver, kidneys, etc., or mental disorders; (III) patients with endocrine diseases and metabolic diseases, such as diabetes, thyroid disorders, etc.; (IV) patients who were long-term users of medications, including sedatives, antidepressants, anticholinergic drugs, antipsychotic drugs, and calcium channel blocker agents; (V) patients with muscular diseases (Figure 1).

\section{Study methods}

In the traditional medical treatment group, the patients 
received traditional medical treatment in the form of biological feedback and drug therapy. Biological feedback therapy consisted of pelvic floor muscle training and functional electrical stimulation were performed alternately once daily, for 30 minutes each time and for continuous 3 courses, with 10 days as 1 course. For the drug therapy, patients were given polycarbophil calcium tablets (State Food and Drug Administration approval no. H20110109; Manufacturer: Suzhou CHUNG-HWA Chemical and Pharmaceutical Industrial Co., Ltd.; Specification: $0.5 \mathrm{~g} \times$ $6 \mathrm{~s} \times 2$ plates), $1 \mathrm{~g}$ taken orally each time, 3 times daily for 1 month.

In the surgical treatment group, patients underwent internal sphincterotomy of the anus. Routine blood pressure measurement, routine blood tests, electrocardiogram, hepatorenal function tests, and chest examination were performed preoperatively. After the delivery of sacral nerve anesthesia, the internal anal sphincter pressure was measured to ascertain whether the internal sphincter had developed spasm. Then, a 10-mm-diameter hole was made at the lower border of the umbilicus, and a small hole measuring $5-10 \mathrm{~mm}$ in diameter was made near the McBurney's points in the left and right lower abdomen, after which the surgical instruments were placed. Subsequently, $\mathrm{CO}_{2}$ pneumoperitoneum was established, with pressure maintained at $13-15 \mathrm{mmHg}$. A central approach was chosen, and the inferior mesenteric arteries and veins were treated. To ensure that the rectum remained intact, it was sufficiently freed along the Denonvillier' fascia in the anterior rectal space and the Waldeyer's fascia in the posterior rectal space. Full protection of the pelvic region and the ureteral nerves needed to be ensured throughout the surgical procedure; during the upward separation of the internal and external sphincters, the surgeon incised the internal sphincter in the junction between the levator ani muscle and the rectum, and parts of the internal sphincter tissues were collected for examination. After surgery, the patients followed up as outpatients to allow the treatment effects to be observed at regular intervals.

\section{Observation indices}

Efficacy (primary outcome) evaluated as follows: (I) marked effectiveness was considered when the patient: could defecate smoothly once every 2 days after treatment, with shaped, soft, and voluminous stools, and a significantly shortened defecation time; had normal sleep and diet, and a positive mood; or did not experience recurrence for half a year after the discontinuation of drug therapy. (II) Effectiveness was considered when the patient: could defecate once every 3 or 4 days, more smoothly than before, with improved symptoms and softer stools than before treatment, and a reduced defecation time; or experienced recurrence after discontinuation of drug therapy for half a year. (III) Ineffectiveness was considered when the patient: showed no improvement in symptoms compared with before treatment; could defecate smoothly after taking medication; or experienced recurrence within half a year of discontinuing drug therapy.

The severity of constipation in the 2 groups was evaluated at different time points using the Constipation Scoring System (CSS) (8) (second outcome), which comprises 8 scoring items, with a total score of 30 points. The higher the score, the severer the constipation. Time points were set at 3, 6, and 12 months after treatment (referred to as T1, $\mathrm{T} 2$, and $\mathrm{T} 3$, respectively).

Patients' sleep quality before and after treatment was evaluated using the Pittsburgh Sleep Quality Index (9) (PSQI) (second outcome), which includes 7 scoring items, with a total score of 21 points. The higher the score, the poorer the sleep quality. Anxiety and depression before and after treatment in both groups was evaluated using the Hospital Anxiety and Depression Scale (10) (HADS). Each item in the scale is given a score from $0-3$ points, and the total score ranges from $0-45$ points, with $0-7$ points representing no symptoms, $8-10$ points indicating possible anxiety and depression, 11-21 points indicating anxiety and depression, and $\geq 22$ points indicating severe anxiety and depression.

An anorectal pressure detector (Manufacturer: Shanghai Hanfei Medical Instrument Co., Ltd.; Model: ZGJ-D2) (primary outcome) was used for the evaluation of anal systolic pressure, rectal resting pressure, and rectal defecation pressure before and after treatment in the 2 groups. Patients were told to empty their stools before the test and to contract their anus for the measurement of the anal systolic pressure. Then, the balloon was pushed into rectum ampulla to measure the rectal resting pressure, and the patients were asked to simulate defecation to measure the rectal defecation pressure.

Electromyographic indices of abdominal muscles, such as $10 \mathrm{~s}$ contraction amplitude (10SC), $10 \mathrm{~s}$ relaxation pressure (10SR), and static pressure (net), were compared between the 2 groups before and after treatment (second outcome).

For the balloon expulsion test, the anterior end of a urinary catheter was inserted into the balloon, and the end of the balloon was tightened with threads. Then, the 
Table 2 Comparison of clinical efficacy between the 2 groups [n (\%)]

\begin{tabular}{lccccc}
\hline Group & $\mathrm{n}$ & Marked effectiveness & Effectiveness & Ineffectiveness & Total effective rate of treatment \\
\hline Surgical treatment group & 125 & $76(60.80 \%)$ & $38(30.40 \%)$ & $11(8.80 \%)$ & $91.20 \%(114 / 125)$ \\
Traditional medical & 125 & $54(43.20 \%)$ & $46(36.80 \%)$ & $25(20.00 \%)$ & $80.00 \%(100 / 125)$ \\
treatment group & & & & 6.360 \\
$\chi^{2}$ & & & & 0.012 \\
$\mathrm{P}$ & & & & \\
\hline
\end{tabular}

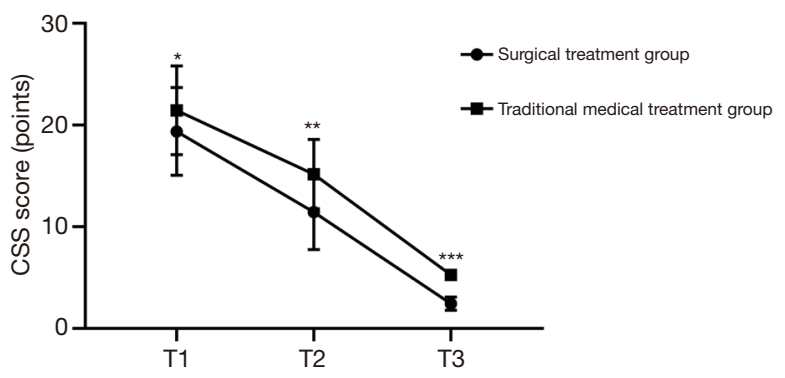

Figure 2 Comparison of CSS scores at different time points after treatment between the 2 groups $(\bar{x} \pm \mathrm{s})$. The abscissa represents T1, $\mathrm{T} 2$, and $\mathrm{T} 3$, while the ordinate represents CSS score, points. In the surgical treatment group, the CSS scores at T1, T2 and T3 were $(19.37 \pm 4.32)$ points, $(11.45 \pm 3.69)$ points, and $(2.44 \pm 0.64)$ points, respectively. In the traditional medical treatment group, the CSS scores at T1, T2 and T3 were $(21.44 \pm 4.36)$ points, $(15.17 \pm 3.41)$ points, and $(5.27 \pm 0.25)$ points, respectively. * indicates a significant difference in the CSS scores of the 2 groups at $\mathrm{T} 1(\mathrm{t}=3.771$, $\mathrm{P}=0.000) ;{ }^{* *}$ indicates a significant difference in the CSS scores of the 2 groups at $\mathrm{T} 2(\mathrm{t}=8.278, \mathrm{P}=0.000) ;{ }^{* * *}$ indicates a significant difference in the CSS scores of the 2 groups at T3 $(\mathrm{t}=46.049$, $\mathrm{P}=0.000)$.

external balloon was soaked in paraffin oil for lubrication, placed in the patient's rectum ampulla, and then $50 \mathrm{~mL}$ of normothermic air injected. After the closure of the external end of the urinary catheter, the patient was instructed to push the balloon out forcefully in a squatting position, and the ejection time was recorded $(11,12)$. A time within 5 minutes was considered to be negative or normal defecation function, and a time exceeding 5 minutes or failure to eject the balloon was considered as positive or defecation dysfunction (second outcome).

Defecography was performed before and after treatment in both groups (second outcome). The filling and mucosal phases were recorded at the time points of resting, anus contraction, and defecation with force, so as to measure the rectocele, perineum descending distance, and anorectal angle in the effort phase (13).

For patients in the surgical treatment group, the condition of the anus was observed before treatment, at 2 days after surgery, and at 5 days after surgery (second outcome).

\section{Statistical analysis}

All data were processed for statistical analysis using SPSS version 21.0 software (IBM, Armonk, NY, USA), and GraphPad Prism 7 (GraphPad Software, San Diego, USA) was used to visualize data. Measurement data were expressed as $(\bar{x} \pm \mathrm{s})$ and tested using the $\mathrm{t}$ test. Enumeration data were expressed as $[\mathrm{n}(\%)]$ and tested using the $\chi^{2}$ test. Differences were considered to have statistical significance when $\mathrm{P}<0.05$.

\section{Results}

\section{Comparison of clinical efficacy between the 2 groups}

As shown in Table 2, the total effective rate of treatment in the surgical treatment group was significantly higher than that in the traditional medical treatment group $(\mathrm{P}<0.05)$.

\section{Comparison of the CSS scores of the 2 groups at different time points after treatment}

At T1, T2, and T3, the CSS scores were significantly reduced in the surgical treatment group compared to the traditional medical treatment group $(\mathrm{P}<0.05)$, as shown in Figure 2.

\section{Comparison of the HADS and PSQI scores of the 2 groups before and after treatment}

As shown in Figure 3, the HADS and PSQI scores in the 2 


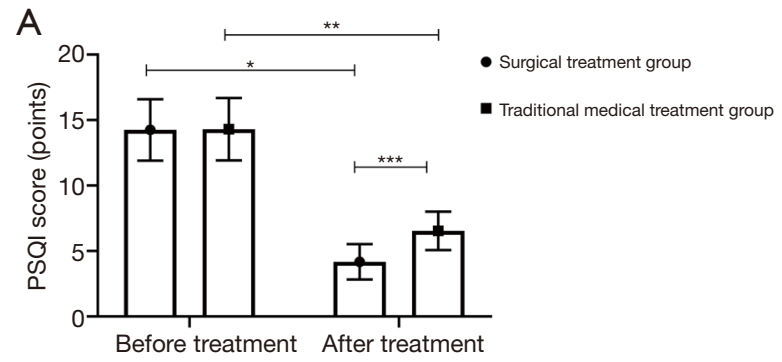

B

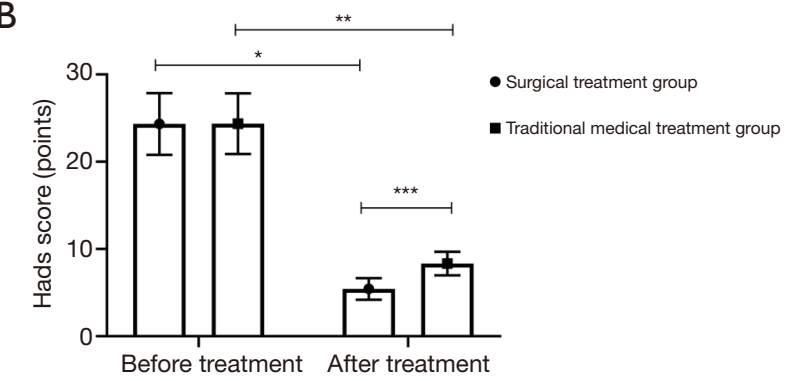

Figure 3 Comparison of the HADS and PSQI scores of the 2 groups before and after treatment $(\bar{x} \pm \mathrm{s})$. (A) A comparison of the PSQI scores of the 2 groups before and after treatment; the abscissa represents before and after treatment, and the ordinate represents PSQI points. In the surgical treatment group, the PSQI scores before and after treatment were $(14.25 \pm 2.34)$ points and $(4.17 \pm 1.35)$ points, respectively. In the traditional medical treatment group, the PSQI scores before and after treatment were $(14.31 \pm 2.38)$ points and $(6.54 \pm 1.47)$ points, respectively. * indicates a significant difference in the PSQI score before and after treatment in the surgical treatment group $(\mathrm{t}=41.717, \mathrm{P}=0.000)$; ** indicates a significant difference in the PSQI score before and after treatment in the traditional medical treatment group $(\mathrm{t}=31.055, \mathrm{P}=0.000)$; *** indicates a significant difference in the PSQI scores of the 2 groups after treatment $(\mathrm{t}=13.276, \mathrm{P}=0.000)$. (B) A comparison of the HADS scores of the 2 groups before and after treatment; the abscissa represents before and after treatment, and the ordinate represents HADS points. In the surgical treatment group, the HADS scores before and after treatment were $(24.33 \pm 3.54)$ points and $(5.43 \pm 1.24)$ points, respectively. In the traditional medical treatment group, the HADS scores before and after treatment were $(24.36 \pm 3.48)$ points and $(8.34 \pm 1.35)$ points, respectively. ${ }^{*}$ indicates a significant difference in the HADS scores before and after treatment in the surgical treatment group $(\mathrm{t}=56.335, \mathrm{P}=0.000) ;$ ** indicates a significant difference in the HADS scores before and after treatment in the traditional medical treatment group $(\mathrm{t}=47.984, \mathrm{P}=0.000)$; ${ }^{* * *}$ indicates a significant difference in the HADS scores of the 2 groups after treatment $(\mathrm{t}=17.749, \mathrm{P}=0.000)$. HADS, Hospital Anxiety and Depression Scale; PSQI, Pittsburgh Sleep Quality Index. groups were significantly lower after treatment than before treatment $(\mathrm{P}<0.05)$. After treatment, the HADS and PSQI scores in the surgical treatment group were significantly lower than those in the traditional medical treatment group $(\mathrm{P}<0.05)$.

\section{Comparison of anorectal pressure in the 2 groups before and after treatment}

As shown in Table 3, no significant differences were found in anal systolic pressure, rectal defecation pressure, or rectal resting pressure between the 2 groups before treatment $(\mathrm{P}>0.05)$. After treatment, the anal systolic pressure and rectal defecation pressure were both higher in the surgical treatment group than in the traditional medical treatment group $(\mathrm{P}<0.05)$, while the rectal resting pressure was significantly lower in the surgical treatment group than in the traditional medical treatment group $(\mathrm{P}<0.05)$.

\section{Comparison of abdominal muscle electromyographic indices in the 2 groups before and after treatment}

As shown in Table 4, before treatment, no significant differences existed between the 2 groups in terms of 10SC, 10SR, or net $(\mathrm{P}>0.05)$. After treatment, the 10SC and net in the surgical treatment group were significantly higher than those in the traditional medical treatment group $(\mathrm{P}<0.05)$, whereas the 10SR was significantly lower in the surgical treatment group than in the traditional medical treatment group $(\mathrm{P}<0.05)$.

\section{Comparison of the negative conversion rate with the balloon expulsion test between the 2 groups}

Regarding the balloon expulsion test, there were 94 positive cases before treatment and 13 positive cases after treatment in the surgical treatment group, with a negative conversion rate of $86.17 \%$. In the traditional medical treatment group, there were 92 positive cases before treatment and 24 positive cases after treatment, with a negative conversion rate of $73.91 \%$. The negative conversion rate of balloon expulsion test differed significantly between the 2 groups after treatment $\left(\chi^{2}=4.384, \mathrm{P}=0.036\right)$.

\section{Comparison of defecographic indices in the 2 groups before and after treatment}

As shown in Table 5, no significant differences were 
Table 3 Comparison of anorectal pressure in the 2 groups before and after treatment $\left(\bar{x}_{ \pm \mathrm{s}}, \mathrm{mmHg}\right)$

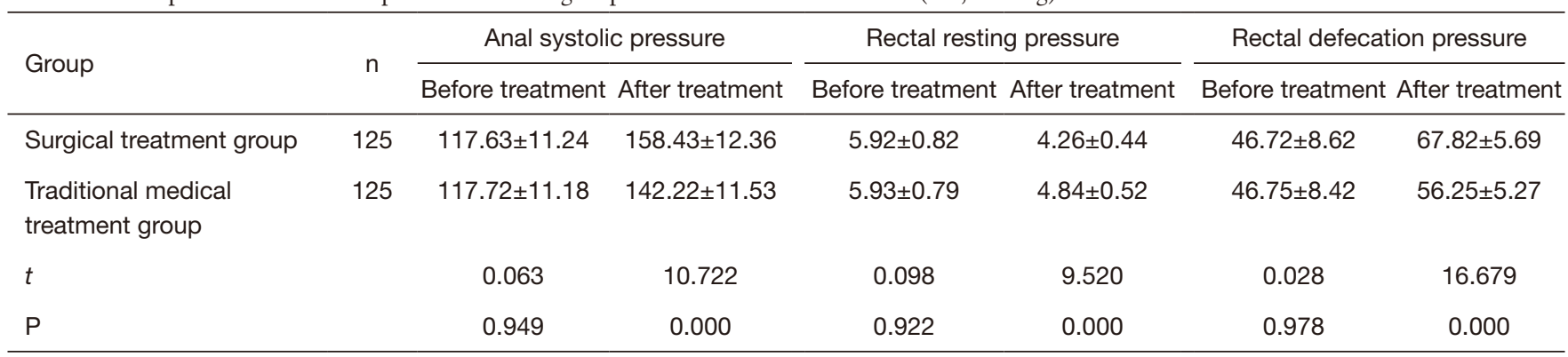

Table 4 Comparison of abdominal muscle electromyographic indices in the 2 groups before and after treatment $(\bar{x} \pm s)$

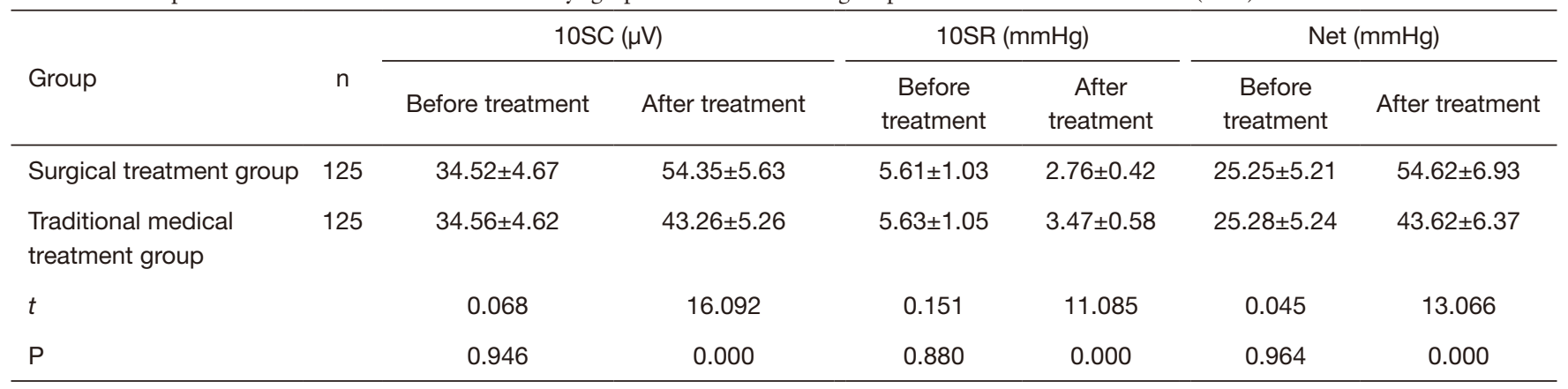

Table 5 Comparison of defecographic indices in the 2 groups before and after treatment $\left(\bar{x}_{ \pm} \mathrm{S}\right)$

\begin{tabular}{|c|c|c|c|c|c|c|c|}
\hline \multirow{2}{*}{ Group } & \multirow{2}{*}{$\mathrm{n}$} & \multicolumn{2}{|c|}{ Rectocele (mm) } & \multicolumn{2}{|c|}{$\begin{array}{l}\text { Perineum descending } \\
\text { distance }(\mathrm{mm})\end{array}$} & \multicolumn{2}{|c|}{$\begin{array}{l}\text { Anorectal angle of efforts } \\
\text { phase after treatment }\left(^{\circ}\right)\end{array}$} \\
\hline & & Before treatment & After treatment & $\begin{array}{l}\text { Before } \\
\text { treatment }\end{array}$ & $\begin{array}{c}\text { After } \\
\text { treatment }\end{array}$ & $\begin{array}{l}\text { Before } \\
\text { treatment }\end{array}$ & After treatment \\
\hline Surgical treatment group & 125 & $23.42 \pm 1.74$ & $2.15 \pm 0.27$ & $44.73 \pm 3.26$ & $10.25 \pm 2.62$ & $134.26 \pm 8.72$ & $118.15 \pm 5.62$ \\
\hline Traditional medical treatment group & 125 & $23.59 \pm 1.68$ & $2.76 \pm 0.36$ & $44.81 \pm 3.18$ & $12.25 \pm 2.41$ & $134.29 \pm 8.69$ & $122.83 \pm 5.48$ \\
\hline$P$ & & 0.433 & 0.000 & 0.845 & 0.000 & 0.978 & 0.000 \\
\hline
\end{tabular}

observed between the 2 groups in terms of the rectocele, perineum descending distance, or anorectal angle in the effort phase before treatment $(\mathrm{P}>0.05)$. After treatment, the rectocele, perineum descending distance, and anorectal angle in the effort phase were significantly lower in the surgical treatment group than in the traditional medical treatment group $(\mathrm{P}<0.05)$.

\section{Comparison of anal bealing in the surgical treatment group before and after treatment}

Representative images of anal healing in a patient in the surgical treatment group before and after treatment are shown in Figure 4A-4C.

\section{Discussion}

With the dietary changes and increase in life pressure seen in recent years, the number of patients with constipation has been rising. Constipation is often complicated by anal fissure, hemorrhoids, and other colorectal diseases, which increases the risk of colorectal cancer $(14,15)$. Most western medical treatments are based on drug therapy, biological feedback, and improved dietary structure. Although such 

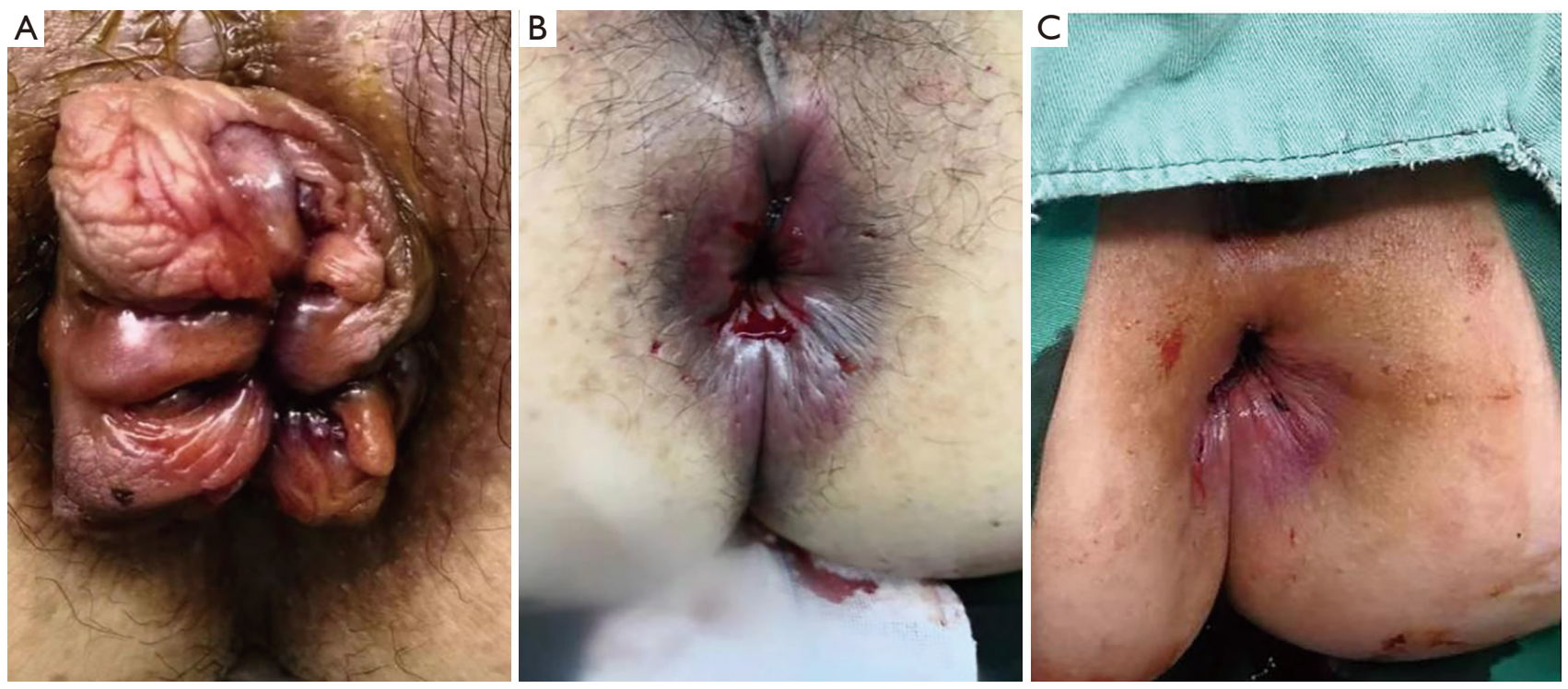

Figure 4 Anal healing of a patient in the surgical treatment group before and after treatment (A) The patient's anal condition before treatment; (B) anal healing at 2 days after surgery; (C) anal healing at 5 days after surgery.

therapeutic approaches are effective, they cannot radically remove diseases and also stimulate the intestinal tract to a certain extent, which can easily lead to intestinal flora disturbance and increase the difficulty of treatment (16).

Outlet obstructive and mixed constipation are common forms of constipation, whose patients often present with intractable difficulty in defecating, long defecation times, and a feeling of incomplete evacuation after defecation. At the same time, these patients also suffer from psychological disorders, which seriously affect their quality of life. Clinical studies have confirmed (17) that under some pathological conditions, the internal sphincter undergoes spasm and hypertrophy in response to inflammatory stimulation. This leads to abnormal myogenic and neurogenic pressure changes as well as failure to effectively perform contraction and relaxation at rest or during defecation, thus affecting the coordination of internal and external sphincter function and subsequently resulting in defecation disorders. Therefore, the application of internal sphincterotomy of the anus for this cohort of patients can effectively correct the paradoxical movement of the internal and external sphincters, improve persistent spasm caused by internal sphincter lesions, correct the allocation of neurogenic pressure to the internal anal sphincters, and improve the coordination of internal and external anal sphincter movement, thus restoring normal intestinal motility and defecation function (18-20).

The CSS score can effectively be used to determine whether patients have constipation, as well as the severity of their constipation. In this study, we applied traditional medical treatment or internal anal sphincterotomy to patients with outlet obstructive and mixed constipation, in our hospital. The CSS scores in both groups showed a decreasing trend at 3, 6, and 12 months after treatment, and those in the surgical treatment group were significantly lower than those in the traditional medical treatment group at different time points $(\mathrm{P}<0.001)$. These results suggest that surgical treatment modalities can significantly relieve patients' severity of constipation and the symptom of difficulty in defecation, thus effectively promoting treatment effect.

Defecography, which involves the injection of contrast agents into the patient's rectum and carrying out active and static observations during defecation, can clearly show functional and organic lesions in the rectum and the anal canal. It can therefore provide a basis for the clinical diagnosis and treatment of each type of constipation $(21,22)$. Our study confirmed that all defecographic indices were significantly lower in the surgical treatment group than in the traditional medical treatment group after treatment $(\mathrm{P}<0.001)$. Through comparison of anal healing of a patient in the surgical treatment group before treatment, at 2 days after surgery, and at 5 days after surgery, this study found that the patient had severe anal fissure before treatment and a small amount of anal bleeding at 2 days after surgery; 
at 5 days after surgery, the anus showed signs of gradual healing (as shown in Figure 3). These observations indicate that internal sphincterotomy of the anus can effectively shorten patients' recovery time and facilitate the recovery of defecation function. Anorectal tonometry can be used to reveal the potential dysfunction of intestinal muscles or nerves, accurately assess coordinated pelvic floor muscle contractions during bowel movements, and examine rectal sensory thresholds and changes in rectal response reflexes $(23,24)$. This study also demonstrated that after treatment, the anal systolic pressure and rectal defecation pressure were both higher in the surgical treatment group than in the traditional medical treatment group $(\mathrm{P}<0.05)$. In their study, Gilsenan et al. (25) reported that after internal sphincterotomy of the anus in patients with low rectal cancer, the anal systolic pressure was $(145.63 \pm 8.94) \mathrm{mmHg}$, which was significantly higher than the $(113.24 \pm 7.46)$ $\mathrm{mmHg}$ measured before treatment, suggesting that internal anal sphincterotomy can increase anal systolic pressure and improve the prognosis of the patients with colorectal diseases.

In conclusion, internal sphincterotomy of the anus can significantly alleviate defecation disorders, eliminate adverse emotions, and improve clinical symptoms in patients with outlet obstructive and mixed constipation. Therefore, this surgical procedure is worthy of clinical application and promotion.

\section{Acknowledgments}

Funding: This study was supported by the Chongqing Municipal Health and Health Committee ([2014] No. 56).

\section{Footnote}

Reporting Checklist: The authors have completed the CONSORT reporting checklist. Available at https://dx.doi. org/10.21037/apm-21-1190

Trial Protocol: Available at https://dx.doi.org/10.21037/apm21-1190

Data Sharing Statement: Available at https://dx.doi. org/10.21037/apm-21-1190

Conflicts of Interest: All authors have completed the ICMJE uniform disclosure form (available at https://dx.doi. org/10.21037/apm-21-1190). The authors have no conflicts of interest to declare.

Ethical Statement: The authors are accountable for all aspects of the work in ensuring that questions related to the accuracy or integrity of any part of the work are appropriately investigated and resolved. All procedures performed in this study involving human participants were in accordance with the Declaration of Helsinki (as revised in 2013). This study was approved by the ethics committee of the People's Hospital of Dazu (Chongqing, China) (No.20140456), and all patients signed informed consent.

Open Access Statement: This is an Open Access article distributed in accordance with the Creative Commons Attribution-NonCommercial-NoDerivs 4.0 International License (CC BY-NC-ND 4.0), which permits the noncommercial replication and distribution of the article with the strict proviso that no changes or edits are made and the original work is properly cited (including links to both the formal publication through the relevant DOI and the license). See: https://creativecommons.org/licenses/by-nc-nd/4.0/.

\section{References}

1. Mungovan SF, Carlsson SV, Gass GC, et al. Preoperative exercise interventions to optimize continence outcomes following radical prostatectomy. Nat Rev Urol 2021;18:259-81.

2. Dias NT, Ferreira LR, Fernandes MG, et al. A Pilates exercise program with pelvic floor muscle contraction: Is it effective for pregnant women? A randomized controlled trial. Neurourol Urodyn 2018;37:379-84.

3. Martinez-Ochoa MJ, Fernandez-Dominguez JC, MoralesAsencio JM, et al. Effectiveness of an Osteopathic Abdominal Manual Intervention in Pain Thresholds, Lumbopelvic Mobility, and Posture in Women with Chronic Functional Constipation. J Altern Complement Med 2018;24:816-24.

4. Mosalanejad F, Afrasiabifar A, Zoladl M. Investigating the combined effect of pelvic floor muscle exercise and mindfulness on sexual function in women with multiple sclerosis: a randomized controlled trial. Clin Rehabil 2018;32:1340-7.

5. Zheng S, Yao J, Chinese Geriatric Society EBoCJoG. Expert consensus on the assessment and treatment of chronic constipation in the elderly. Aging Med (Milton) 2018;1:8-17.

6. Chedid V, Vijayvargiya P, Halawi H, et al. Audit of 
the diagnosis of rectal evacuation disorders in chronic constipation. Neurogastroenterol Motil 2019;31:e13510.

7. Sobhgol SS, Priddis H, Smith CA, et al. The Effect of Pelvic Floor Muscle Exercise on Female Sexual Function During Pregnancy and Postpartum: A Systematic Review. Sex Med Rev 2019;7:13-28.

8. Yiannakou Y, Etherson K, Close H, et al. A randomized double-blinded sham-controlled cross-over trial of tined-lead sacral nerve stimulation testing for chronic constipation. Eur J Gastroenterol Hepatol 2019;31:653-60.

9. Aziz I, Whitehead WE, Palsson OS, et al. An approach to the diagnosis and management of Rome IV functional disorders of chronic constipation. Expert Rev Gastroenterol Hepatol 2020;14:39-46.

10. Virtuoso JF, Menezes EC, Mazo GZ. Effect of Weight Training with Pelvic Floor Muscle Training in Elderly Women with Urinary Incontinence. Res Q Exerc Sport 2019;90:141-50.

11. Jiang Y, Tang Y, Lin L. Clinical Characteristics of Different Primary Constipation Subtypes in a Chinese Population. J Clin Gastroenterol 2020;54:626-32.

12. Southwell BR. Treatment of childhood constipation: a synthesis of systematic reviews and meta-analyses. Expert Rev Gastroenterol Hepatol 2020;14:163-74.

13. Daly D, Cusack C, Begley C. Learning about pelvic floor muscle exercises before and during pregnancy: a crosssectional study. Int Urogynecol J 2019;30:965-75.

14. Mugie SM, Koppen IJN, van den Berg MM, et al. Brain processing of rectal sensation in adolescents with functional defecation disorders and healthy controls. Neurogastroenterol Motil 2018;30.

15. Alawady M, Emile SH, Abdelnaby M, et al. Posterolateral versus lateral internal anal sphincterotomy in the treatment of chronic anal fissure: a randomized controlled trial. Int J Colorectal Dis 2018;33:1461-7.

16. Keller AO, Ortiz A. Physical Activity Health Communication for Adults With Mood Disorders in the

Cite this article as: Bin C, Gong D, He Y. A randomised controlled study: the effects of internal sphincterotomy of the anus on defecation disorders in patients with outlet obstructive and mixed constipation. Ann Palliat Med 2021;10(8):8718-8727. doi: 10.21037/apm-21-1190
United States. West J Nurs Res 2020;42:97-107.

17. Olajossy M, Olajossy B, Wnuk S, et al. Blood serum concentrations of kynurenic acid in patients diagnosed with recurrent depressive disorder, depression in bipolar disorder, and schizoaffective disorder treated with electroconvulsive therapy. Psychiatr Pol 2017;51:455-68.

18. Mayer O, Jr., Bruthans J, Seidlerova J, et al. Mood disorders impaired quality of life but not the mortality or morbidity risk in stable coronary heart disease patients. Acta Cardiol 2019. [Epub ahead of print]. doi: 10.1080/00 015385.2019 .1653568 .

19. Kashanian M, BozorgzadehVostaKolaei S, Naderi Z, et al. Evaluation of the relationship between serum anti TPO antibody levels and mood disorders in euthyroid pregnant women. J Matern Fetal Neonatal Med 2020;33:3233-7.

20. Pelletier L, O'Donnell S, Dykxhoorn J, et al. Underdiagnosis of mood disorders in Canada. Epidemiol Psychiatr Sci 2017;26:414-23.

21. Stange JP, Jenkins LM, Pocius S, et al. Using resting-state intrinsic network connectivity to identify suicide risk in mood disorders. Psychol Med 2020;50:2324-34.

22. Miller L, Shade M, Vasireddy V. Beyond screening: assessment of perinatal depression in a perinatal care setting. Arch Womens Ment Health 2009;12:329-34.

23. Resende APM, Bernardes BT, Stupp L, et al. Pelvic floor muscle training is better than hypopressive exercises in pelvic organ prolapse treatment: An assessor-blinded randomized controlled trial. Neurourol Urodyn 2019;38:171-9.

24. Schindler S, Schmidt L, Stroske M, et al. Hypothalamus enlargement in mood disorders. Acta Psychiatr Scand 2019;139:56-67.

25. Gilsenan A, Fortuny J, Cainzos-Achirica M, et al. Cardiovascular Safety of Prucalopride in Patients with Chronic Constipation: A Multinational Population-Based Cohort Study. Drug Saf 2019;42:1179-90.

(English Language Editor: J. Reynolds) 